\title{
Helical Compounds Forming Gas-Phase Dimers: A Dispersion-corrected Density Functional Investigation
}

\author{
Pornthip Tongying, Thanasat Sooksimuang, ${ }^{\dagger}$ and Yuthana Tantirungrotechai* \\ National Nanotechnology Center, National Science and Technology Development Agency, Pathumthani, 12120 Thailand \\ *E-mail: yuthana@nanotec.or.th \\ ${ }^{\dagger}$ National Metal and Materials Technology Center, National Science and Technology Development Agency, \\ Pathumthani, 12120 Thailand \\ Received September 27, 2010, Accepted February 12, 2011
}

\begin{abstract}
Chiral discrimination is the ability to distinguish one enantiomeric form over another. The differential binding interaction between two molecules with the same helicity and those with the opposite helicity was investigated by using dispersion-corrected density functional theory. [5]helicene, tetrahydro[5]helicene and the polar D- $\pi$ A compounds, 3,12-dimethoxy-7,8-dicyano-[5]helicene and 3,12-dimethoxy-7,8-dicyano-tetrahydro[5]helicene were the monomers considered in this study. In gas phase, the dimeric interaction from two helical molecules with the opposite handedness is greater than from those with the same handedness. The stable configurations of such dimers were identified. The most stable configuration tends to be the one with maximum contact between monomers.
\end{abstract}

Key Words : Helicene, Dispersion-corrected density functional, Aggregate, Chiral discrimination

\section{Introduction}

Chirality is a very fundamental concept in molecular science. ${ }^{1-5}$ In chemistry, chirality usually arises from having chiral center, leading to a three-dimensional structure with a non-superimposible mirror image. The identification of the chiral configuration is very important because compounds with opposite chirality can have different biological activities. The receptor site built from stereospecific molecular unit binds with one chiral form of ligand rather than another due to their complementarity. ${ }^{3-5}$ Such behavior is called chiral discrimination.

Helical structures exhibit chirality although they do not possess a chiral center. This is called helical chirality. Helical structures with right-handed or left-handed helicity are denoted $\mathrm{P}$ - and $\mathrm{M}$-congurations, respectively. ${ }^{2,6}$ In this work, we investigated the differential binding interactions between gas-phase dimers formed from two monomers with the same helicity and from those with the opposite helicity. We selected [5]helicene and its derivatives as model compounds for our study.

Helicene is a chiral ortho-annulated $\pi$-conjugated system. ${ }^{7}$ Due to its helical structure and its conjugated nature, researchers are exploring its outstanding chirooptical properties for use as functional materials in many areas including nonlinear optics and organic light-emitting diode applications. $^{8-11}$ The helicene framework can be modified by incorporating other chemical elements or substituents. ${ }^{12-15}$ Sahasithiwat et al. modified the [5] helicene framework by incorporating electron donor and acceptor groups. ${ }^{16}$ The resulting D- $\pi$-A compound, 3,12-dimethoxy-7,8-dicyano[5]helicene, is used as an emissive material for organic light emitting diodes. We include this compound in our study in addition to [5] helicene (see Fig. 1(a)) due to its polar nature. We also consider tetrahydro[5] helicene and 3,12-dimethoxy7,8-dicyano-tetrahydro[5]helicene which possess a carboncarbon single bond on the $2^{\text {nd }}$ and $4^{\text {th }}$ rings of [5] helicene in this study (see Fig. 1(b)). These two compounds should have a smaller $\pi-\pi$ interaction than the original helicene due to partial hydrogenation of the helicene framework.

Studying weakly interacting systems by quantum mechanical models can be a challenging task. In the case of conventional $a b$ initio methods, at least the second-order MollerPlesset perturbation theory (MP2) method which include electron correlation is required for a proper description of such systems. ${ }^{17,18}$ This approach is too expensive to be practical for large systems like [5]helicene dimers. An alternative approach is to use the density functional theory (DFT) which is nowadays the method of choice for theoretical investigation of chemical systems. However, even the well-known B3LYP hybrid functional is not appropriate for treating weak interactions because of its incorrect long-range behavior in the exchange-correlation potential. ${ }^{19}$ Many novel exchange-correlation functionals, such as the CAM-B3LYP ${ }^{20}$ and the Minnesota M06 and M08 functionals, ${ }^{19,21-23}$ have been proposed to overcome this problem. Alternatively, a practical approach is to include an empirical dispersioncorrected term as originally proposed by Ahlrichs ${ }^{24}$ and developed further for DFT by Grimme..$^{25,26}$ The dispersioncorrected density functional theory (DFT-D) is a very convenient approach. This method is well suited not only for treating weak interaction but also for studying chemical reactions such as isomerization reactions. ${ }^{27}$

Grimme et al. provide a clear perspective on the applications of dispersion-corrected density functional theory for supramolecular structures, aggregates and complexes of 


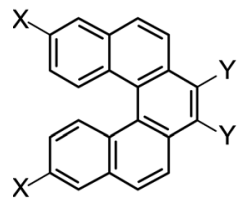

(a)

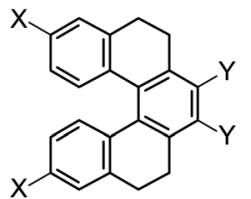

(b)
Figure 1. The molecular structure of [5]helicene and tetrahydro [5]helicene monomers and their polar 3,12-dimethoxy-7,8-dicyano derivatives. (a) [5]helicene, $\mathrm{X}, \mathrm{Y}=\mathrm{H} ; \mathrm{3}$,12-dimethoxy-7,8-dicyano[5]helicene, $\mathrm{X}=\mathrm{OMe}, \mathrm{Y}=\mathrm{CN}$. (b) Tetrahydro-[5]helicene, $\mathrm{X}, \mathrm{Y}=\mathrm{H}$; 3,12-dimethoxy-7,8-dicyano-tetrahydro-[5]helicene, $\mathrm{X}=\mathrm{OMe}, \mathrm{Y}=\mathrm{CN}$.

organic molecules. ${ }^{27}$ Mackie and DiLabio applied the DFTD method to study interactions of several large polyaromatic hydrocarbon dimers. In their implementation, the authors optimized the carbon atom-centered effective core-type potential to correct the long-range behavior of the exchangecorrelation potential. Good agreement in the structure and binding energy with high-level benchmark data was observed. ${ }^{28}$ Moellmann and Grimme investigated the dispersion effect for predicting the molecular crystal packing of a bis-thiophene derivative. ${ }^{29}$ The dispersion-corrected DFT model predicts the crystal packing effect, the gas-phase structure, and the lattice energy of RESVAN, a bis-thiophene derivative, in good agreement with high-quality quantum chemical methods. However, they observed a near cancellation of intramolecular and intermolecular dispersion effects in the solid state structure. This results in the dispersion-uncorrected DFT model yielding a more reasonable solid state structure than the dispersion-corrected DFT model.

We selected the DFT-D model for calculating the differential binding interaction between $\mathrm{M}-\mathrm{M}$ and $\mathrm{P}-\mathrm{M}$ dimers constructed from [5] helicene and its derivatives (see Fig. 1). Our objectives are two-fold. First, several possible binding configurations of gas-phase dimers are identified. Second, the effect of structural modifications on the binding mode of these compounds is investigated. By comparing the most energetically stable form of the M-M and P-M dimers, some information on chiral discrimination of such compounds in gas-phase could be deduced.

There have been several reports on the chiral discrimination of helical structures including a recent review by Amemiya and Yamaguchi. ${ }^{6,30,31}$ Yamaguchi et al. studied the chemistry of 1,12-dimethylbenzo[c]phenanthrene as a chiral building block. ${ }^{32}$ They concluded from several studies that one helix prefers another helix with the same helicity. Conclusions on chiral discrimination are usually drawn from solid state crystal structure, ${ }^{30}$ from folding of helical oligomers or from complex formation with different molecules. ${ }^{31,33}$ Honzawa et al. studied the binding of 1,12-dimethylbenzo [c]phenanthrene, a basic [4]-helicene derivative, to righthanded calf thymus DNA by various spectroscopic and calorimetric techniques. ${ }^{33}$ By estimating the complex formation constant and the binding formation free energy, they observed a higher affinity between calf-thymus DNA with the righthanded $(\mathrm{P})$-helicene than with the left-handed $(\mathrm{M})$-helicene. Interestingly, the enthalpic contribution favors the (M)- helicene more than the $(\mathrm{P})$-helicene by $2.1 \mathrm{kcal} / \mathrm{mol}$. It appears that the entropic contribution results in the $\mathrm{P}$ form being more favorable in binding with calf thymus DNA than the $\mathrm{M}$ form. When accounting for chiral discrimination from crystal structure, there are some other factors such as cooperative hydrogen bonding ${ }^{30}$ that play an important role in determining the crystal packing. Murguly et al. also observed different self-assembly behaviors in the crystal and in solution phases. ${ }^{30}$ To avoid many-body effects, therefore, only the gas-phase aggregates were considered in our study. We consider the differential interaction energy between M$\mathrm{M}$ and P-M gas-phase dimers as an indicator of chiral discrimination.

\section{Methods}

All calculations were performed using the ORCA program. ${ }^{34}$ We consider the gas-phase helical dimers constructed from left-handed (M)-left-handed (M) and right-handed (P)left-handed $(\mathrm{M})$ pairs of monomers. The monomers are [5]helicene, tetrahydro[5]helicene, 3,12-dimethoxy-7,8-dicyano-[5]helicene and 3,12-dimethoxy-7,8-dicyano-tetrahydro-[5]helicene. Several initial configurations of M-M and $\mathrm{P}-\mathrm{M}$ dimers were generated. These include, for example, head-head, head-tail, stack, antistack, slip and perpendicular configurations.

The geometries of dimer configurations were optimized by using the RI-BLYP-D/def2-SV(P) and RI-BP86-D/def2$\mathrm{SV}(\mathrm{P})$ levels of calculation. Due to the large system size, the resolution of identity (RI) approximation was used to speed up the computation. We chose Grimme's implementation of DFT-D which includes an empirical $1 / R^{6}$ term to capture the correct van der Waals dispersion interaction between monomers. ${ }^{25,26}$ After the optimized configurations were identified, single-point energy calculations were performed using the same functional but with a much larger def2-TZVPP basis set $^{35,36}$ and with the double-hybrid B2PLYP-D/TZVP method. ${ }^{37}$ The double-hybrid functional combines the conventional MP2 correction term with a hybrid functional. The Boys and Bernardi counterpoise correction scheme was used to remove the basis set superposition error (BSSE) in the interaction energy. ${ }^{38}$ The optimized dimeric configurations were identified and grouped together in terms of similar converged structures and interaction energies. The results are shown in Tables 1-4. Only the most favorable configurations for each dimer are shown in Figures 2(a)-(d) while all configurations are reported in the Supplementary Information.

\section{Results and Discussion}

Table 1 reports the $\mathrm{M}-\mathrm{M}$ and $\mathrm{P}-\mathrm{M}$ BSSE-corrected interaction energies of the non-polar [5]helicene dimers. Although several configurations were initially generated, the optimized structures fall into some dominant configurations. The M-M [5]helicene dimers fall into stack, antistack and slip configurations. We employed these terms to describe the relative configuration between the two monomers in a loose 


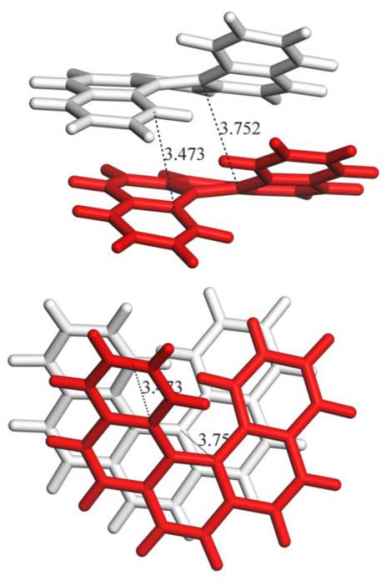

M-M:stack
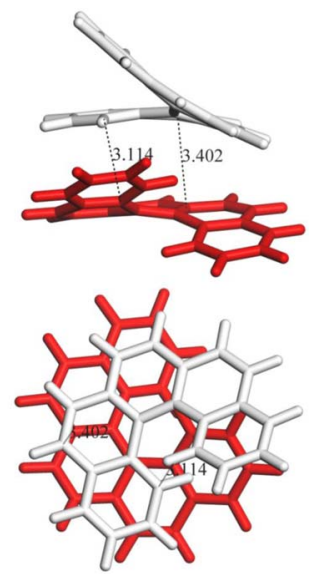

P-M:twist stack

(a) [5]helicene dimer

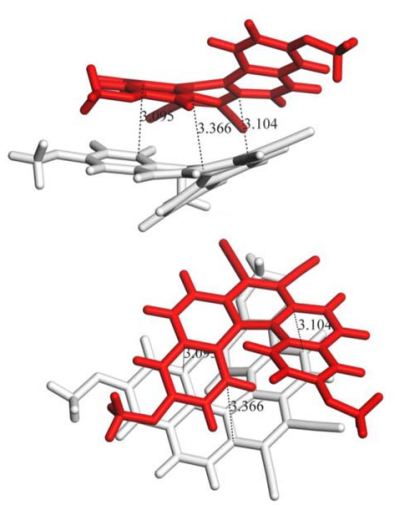

M-M:twist antistack
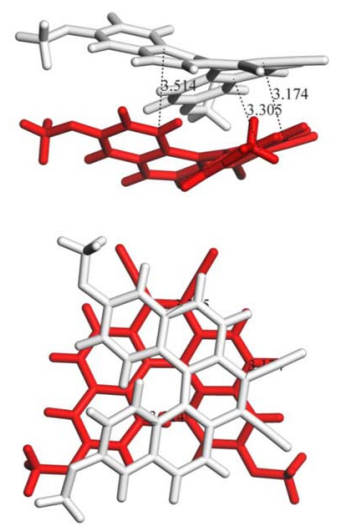

P-M:twist stack

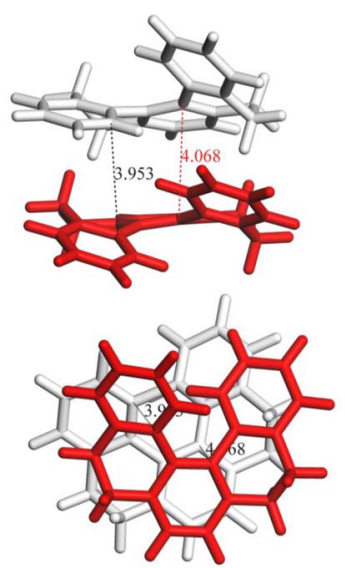

M-M:stack

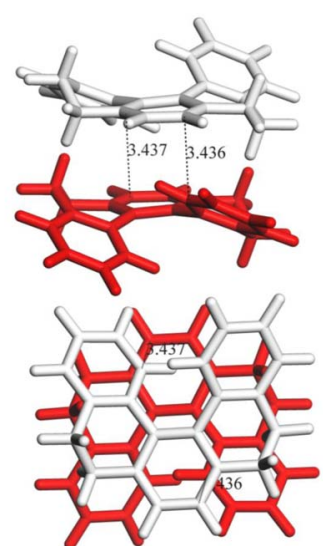

P-M:antistack

(b) tetrahydro[5]helicene dimer

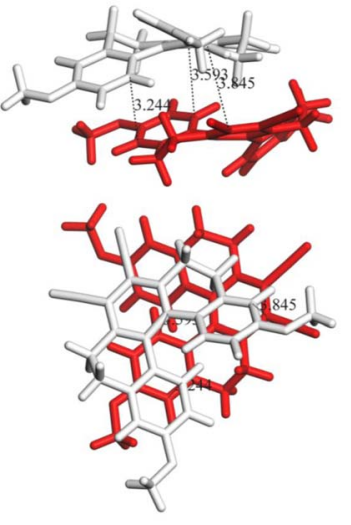

M-M: twist antistack

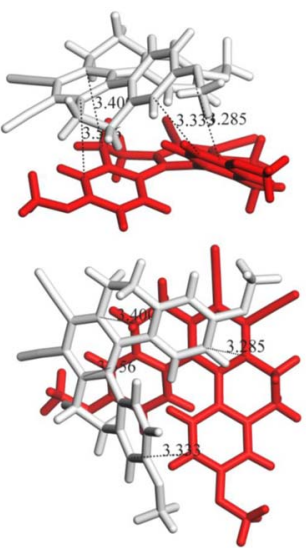

P-M:twist stack

(c) 3,12-dimethoxy-7,8-dicyano-[5]helicene dimer

Figure 2. The most stable M-M and P-M gas phase configuration of (a) [5]helicene (b) tetrahydro[5]helicene (c) 3,12-dimethoxy-7,8dicyano-[5]helicene (d) 3,12-dimethoxy-7,8-dicyano-tetrahydro[5]helicene dimers.

sense. The relative stabilities of the dimeric configurations from all calculation methods considered agree well with one another. The BLYP-D method cannot locate the less important slip configuration while the BP86-D can. However, this does not affect our conclusion because the most stable configuration is the stack configuration, i.e., one monomer above another with the end benzene rings pointing in almost the same direction (see Fig. 2(a)). It is also the configuration

Table 1. The M-M and P-M BSSE-corrected interaction energy (in kcal $/ \mathrm{mol}$ ) of [5]helicene dimers. See Figure 2a for graphical representation

\begin{tabular}{lccccc}
\hline & $\begin{array}{c}\text { BP86-D/ } \\
\text { def2-SV(P) }\end{array}$ & $\begin{array}{c}\text { BP86-D/TZVPP// } \\
\text { BP86-D/def2-SV(P) }\end{array}$ & $\begin{array}{c}\text { BLYP-D/ } \\
\text { def2-SV(P) }\end{array}$ & $\begin{array}{c}\text { BLYP-D/TZVPP// } \\
\text { BLYP-D/def2-SV(P) }\end{array}$ & $\begin{array}{c}\text { B2PLYP-D/TZVP// } \\
\text { BLYP-D/def2-SV(P) }\end{array}$ \\
\hline stack & & M-M & & -13.8 \\
antistack & -15.3 & -16.7 & -13.3 & -14.8 & -12.6 \\
slip & -14.0 & -15.1 & -12.9 & n.a. & n.a. \\
\hline & -10.4 & -11.6 & n.a. & -15.1 & -14.2 \\
twist stack & -15.7 & -16.9 & -14.1 & -13.6 & -13.2 \\
antistack & -14.4 & -15.3 & -12.8 & -13.1 & -12.6 \\
stack & -13.8 & -14.8 & -12.2 & -9.7 & -8.7 \\
slip antistack & -11.6 & -12.6 & -8.8 & -7.9 & -7.1 \\
head-head & -11.4 & -12.6 & -7.7 & & \\
\hline
\end{tabular}


found in the crystal packing. ${ }^{8}$ The antistack configuration which has the end benzene rings pointing in the opposite direction is the next most stable form. Although the energy difference between these two configurations is small, all methods including the definitive B2PLYP-D calculation confirm this trend. Due to the nature of the weak interaction, the interconversion between stack and antistack configurations should be possible. The configuration with one monomer on top of another has an increased contact area between the two monomers and hence there is a greater molecular interaction. To increase the molecular interaction further, the monomer slips slightly relative to the other in both stack and antistack configurations as a result of its nonplanar structure.

The most stable configuration of the P-M [5]helicene dimer is the twist stack configuration. A large degree of relative twist between the two monomers is observed in this configuration (see Fig. 2(a)). The monomer adopts this arrangement to increase the contact area and reduce the intermolecular distance. It turns out that the energetic interaction of the P-M [5]helicene dimer is slightly greater than that of the M-M dimer. The B2PLYP-D model predicts a differential binding energy of $0.4 \mathrm{kcal} / \mathrm{mol}$ This is in line with the observation made by Honzawa et al..$^{33}$ In their complex formation study between a helicene derivative and calf thymus DNA, the enthalpic contribution to the binding constant was shown to favor opposite helicity while including the entropic contribution reversed the conclusion. Other identified stable configurations include antistack, stack, slip antistack and head-head configurations (see Supplementary Information). Considering the stack configuration which is found in crystal packing, the P-M dimer is less favorable than the M-M dimer by about $1.1-1.9 \mathrm{kcal} / \mathrm{mol}$. Our result partly explains why helical compounds crystallize only in the enantiomeric pure form. ${ }^{30}$

Tetrahydro[5]helicene is a molecule with partial hydrogenation. This should reduce the $\pi-\pi$ interaction found in [5]helicene and trigger hydrogen-hydrogen repulsion from saturated carbon atoms in the structure. In the case of the tetrahydro[5]helicene dimer, the M-M dimer prefers the stack configuration while the P-M dimer prefers the antistack configuration (see Table 2 and Fig. 2(b)). Only a marginal difference in interaction energy between M-M and P-M forms is observed in such compounds. The BLYP-D method slightly favors the P-M form over the M-M form while with the BP86-D method this difference is more pronounced.

Comparing the [5]helicene and tetrahydro[5]helicene dimers, both M-M forms favor the stack configuration in which a helical monomer twists in the same direction as the other monomer thus forming another helical twist. On the other hand, the P-M dimer achieves maximum interaction by twisting around its intermolecular axis. For the tetrahydro[5]helicene dimer, the twist leads to the antistack configuration.

For 3,12-dimethoxy-7,8-dicyano-[5]helicene dimer, the M-M form prefers the antistack configuration with a further twist ('twist antistack', see Fig. 2(c)). The BSSE-corrected B2PLYP-D interaction energy is about $-20.4 \mathrm{kcal} / \mathrm{mol}$ which is much greater than that of [5]helicene dimer $(-13.8 \mathrm{kcal} /$ mol) (see Table 3). The methoxy and cyano substituents enhance the electrostatic interaction of the dimer. A simple electrostatic consideration also explains why the monomer twists itself further in such a dimer. This reduces the electrostatic repulsion from substituents of the same type and increases the attraction from substituents of the opposite type. The less stable configurations include stack, twist stack, slip antistack, perpendicular and slip configurations (see Supplementary Information). Table 3 also reports the BSSEcorrected interaction energies for each $\mathrm{P}-\mathrm{M}$ dimeric configuration. The $\mathrm{P}-\mathrm{M}$ dimer prefers the twist stack configuration (see Fig. 2(c)) and has an interaction energy of about -24 $\mathrm{kcal} / \mathrm{mol}$. In terms of differential interaction energy, the P-M gas-phase dimer is more stable than the M-M gas phase dimer.

A similar trend is also observed in 3,12-dimethoxy-7,8dicyano-tetrahydro[5] helicene dimer (see Table 4, and Fig. 2(d)). For the B2PLYP-D method, the binding energy of P-

Table 2. The M-M and P-M BSSE-corrected interaction energy (in kcal/mol) of tetrahydro[5]helicene dimers. See Figure $2 b$ for graphical representation

\begin{tabular}{lccccc}
\hline & $\begin{array}{c}\text { BP86-D/ } \\
\text { def2-SV(P) }\end{array}$ & $\begin{array}{c}\text { BP86-D/TZVPP// } \\
\text { BP86-D/def2-SV(P) }\end{array}$ & $\begin{array}{c}\text { BLYP-D/ } \\
\text { def2-SV(P) }\end{array}$ & $\begin{array}{c}\text { BLYP-D/TZVPP// } \\
\text { BLYP-D/def2-SV(P) }\end{array}$ & $\begin{array}{c}\text { B2PLYP-D/TZVP// } \\
\text { BLYP-D/def2-SV(P) }\end{array}$ \\
\hline stack & & & M-M & & -13.4 \\
antistack & -12.2 & -13.9 & -14.3 & -15.7 & -11.8 \\
slip & -12.3 & -13.7 & -12.2 & -7.2 & -6.8 \\
head-head & -9.3 & -10.4 & -7.2 & -9.2 & -8.4 \\
\hline & -8.4 & -9.4 & -8.7 & & -13.4 \\
\hline antistack & -15.0 & & P-M & -15.2 & -12.6 \\
stack & -14.3 & -16.7 & -14.4 & -14.9 & -10.8 \\
twist stack & -12.3 & -16.1 & -13.9 & -10.9 & -9.2 \\
slip stack & -8.9 & -13.5 & -12.3 & -12.0 & -10.2 \\
head-head & -12.2 & -10.1 & -10.4 & -11.7 & \\
\hline
\end{tabular}


Table 3. The M-M and P-M BSSE-corrected interaction energy (in $\mathrm{kcal} / \mathrm{mol}$ ) of 3,12-dimethoxy-7,8-dicyano-[5]helicene dimers. See Figure $2 \mathrm{c}$ for graphical representation

\begin{tabular}{lccccc}
\hline & $\begin{array}{c}\text { BP86-D/ } \\
\text { def2-SV(P) }\end{array}$ & $\begin{array}{c}\text { BP86-D/TZVPP// } \\
\text { BP86-D/def2-SV(P) }\end{array}$ & $\begin{array}{c}\text { BLYP-D/ } \\
\text { def2-SV(P) }\end{array}$ & $\begin{array}{c}\text { BLYP-D/TZVPP// } \\
\text { BLYP-D/def2-SV(P) }\end{array}$ & $\begin{array}{c}\text { B2PLYP-D/TZVP// } \\
\text { BLYP-D/def2-SV(P) }\end{array}$ \\
\hline twist antistack & \multicolumn{5}{c}{ M-M } \\
stack & -22.3 & -23.8 & -20.5 & -21.8 & -20.4 \\
twist stack & -18.8 & -19.8 & -17.1 & -18.0 & -17.5 \\
slip antistack & -18.5 & -19.7 & -17.3 & -18.0 & -17.0 \\
perpendicular & -21.1 & -22.2 & -15.9 & -17.0 & -15.5 \\
slip & -9.0 & -10.0 & -10.8 & -11.4 & -10.6 \\
\hline & -11.2 & -12.2 & -10.6 & -11.0 & -10.6 \\
\hline twist stack & & & & -24.9 \\
slip antistack & -26.6 & -28 & -24.4 & -20.2 & -19.6 \\
slip stack & -20.7 & -22.1 & -19.1 & -15 & -15.2 \\
stack & -15.1 & -16.2 & -14.1 & -14.7 & -14.2 \\
perpendicular & -13.5 & -14.3 & -14 & -14.6 & -12.8 \\
head-head & -13.1 & -14.7 & -13.7 & -12.2 & -11.2 \\
slip & -12.3 & -12.8 & -12.0 & -10.7 & -9.8 \\
\hline
\end{tabular}

Table 4. The M-M and P-M BSSE-corrected interaction energy (in kcal/mol) of 3,12-dimethoxy-7,8-dicyano-tetrahydro[5]helicene dimers. See Figure $2 \mathrm{~d}$ for graphical representation

\begin{tabular}{lccccc}
\hline & $\begin{array}{c}\text { BP86-D/ } \\
\text { def2-SV(P) }\end{array}$ & $\begin{array}{c}\text { BP86-D/TZVPP// } \\
\text { BP86-D/def2-SV(P) }\end{array}$ & $\begin{array}{c}\text { BLYP-D/ } \\
\text { def2-SV(P) }\end{array}$ & $\begin{array}{c}\text { BLYP-D/TZVPP// } \\
\text { BLYP-D/def2-SV(P) }\end{array}$ & $\begin{array}{c}\text { B2PLYP-D/TZVP// } \\
\text { BLYP-D/def2-SV(P) }\end{array}$ \\
\hline twist antistack & & & M-M & & -21.1 \\
twist stack & -21.4 & -22.8 & -19.8 & -16.5 & -14.1 \\
perpendicular & -15.6 & -17.1 & -15.5 & -16.5 & -14.4 \\
stack & n.a. & n.a. & -15.3 & -15.7 & -13.1 \\
slip antistack & -15.6 & -17.4 & -14.6 & -13.5 & -10.5 \\
slip & n.a. & n.a. & -13.1 & -14.0 & -8.1 \\
head-head & -13.1 & -14.7 & -13.3 & -12.3 & -18.8 \\
\hline & -9.7 & -12.2 & -11.0 & -22.2 & -17.0 \\
\hline twist stack & & & & -20.1 & -13.4 \\
slip antistack & -19.3 & -21.0 & -20.7 & -15.8 & -12.6 \\
stack & -18.9 & -20.8 & -19.0 & -14.5 & -10.0 \\
head-head & -14.9 & -16.6 & -14.7 & -12.5 & -9.7 \\
slip stack & -15.9 & -17.5 & -13.6 & -12.0 & -10.3 \\
side twist & -12.3 & -13.7 & -12.2 & -12.1 & -6.7 \\
slip & -11.2 & -12.7 & -11.3 & -8.6 & \\
perpendicular & n.a. & n.a. & -11.3 & -7.9 & \\
\hline
\end{tabular}

$\mathrm{M}$ dimer is greater than that of the M-M dimer by $-1.1 \mathrm{kcal} /$ mol. The most stable configuration of M-M dimer is the twist stack while the P-M dimer prefers the twist antistack configuration. We observe a lower differential binding interaction between M-M and M-P forms of tetrahydro[5]helicene derivatives than in the case of the helicene derivatives. The enhancement of the interaction energy due to the donor and acceptor substituents is much less pronounced in this tetrahydro[5]helicene derivative than in the [5]helicene derivative. This might be due to the partial hydrogenation in the helicene framework that reduces the $\pi$ electron delocali- zation.

Some less stable configurations observed in this study, such as the head-head configuration, have also been observed experimentally. Murguly et al. synthesized and crystallized the hydrogen-bonded [7]helicene. As a result of the strong hydrogen bonding of the functional groups on the end benzene rings, the head-head dimer is formed as an entity in crystal packing. ${ }^{30}$

The observation that, by considering the differential interaction energy between M-M and P-M forms, a helical molecule prefers to bind with another molecule of opposite 
handedness during gas-phase dimer formation might sound surprising at first. However, as mentioned earlier, several factors, such as the effects of crystal packing, substituents or entropic contributions, might play a role in favoring the binding of helical molecules with the same handedness. Our gas-phase calculation avoids any such influences and should give definitive energetic information on this type of dimer formation.

Finally, after the completion of this work, it came to our attention that favorable binding between two helical molecules with opposite handedness has been reported elsewhere. ${ }^{39}$ $\mathrm{Xu}$ et al. conducted dialysis experiments to obtain the binding formation between thiahetero[7]helicene and lefthanded Z-DNA. The Z-DNA prefers binding with (P)helicene over (M)-helicene. Moreover, right-handed B-DNA converts into left-handed Z-DNA upon binding with (P)helicene. Further investigations on the helicene-DNA systems of Honzawa et al. and $\mathrm{Xu}$ et al. are being carried out in our laboratory.

\section{Conclusions}

The gas-phase dimeric configuration of helicene derivatives were investigated by the DFT-D method to provide information on the differential binding interaction between helical molecules. In the absence of many-body effects, [5]helicene prefers P-M over M-M binding. The P-M dimer adopts the twist stack configuration while M-M favors the stack configuration. On modifying the helicene framework by partial hydrogenation, less differential binding is observed in the tetrahydro[5]helicene dimer. Adding electron withdrawing and donating groups into the helical structure increases the dimeric interaction. In such systems, there is a large twist between monomers. This is to lower electrostatic repulsion and increase electrostatic attraction. Our findings provide energetic information on the chiral discrimination process.

Acknowledgments. We thank the Thailand Research Fund (RSA5180010) and the National Nanotechnology Center (NANOSIM) for financial support. Computational facility at the high performance computing center (HPCC), large scale simulation research laboratory, the National Electronics and Computer Technology Center (NECTEC) is acknowledged. Finally, we thank Dr. Michael A. Allen of Physics Department, Mahidol University for his kind help on proofreading and manuscript editing.

Supplementary Materials Available. This information is available free of charge via the intermet at http://kcsnet.or.kr.

\section{References}

1. Lough, W.; Wainer, I. Chirality in Natural and Applied Science, 1st ed.; Blackwell: 2002.

2. Amouri, H.; Gruselle, M. Chirality in Transition Metal Chemistry:
Molecules, Supramolecular Assemblies and Materials; Wiley: 2009.

3. Meyers, R. A. Encyclopedia of Molecular Biology and Molecular Medicine; VCH Publishers: Weinheim, Germany, 1996.

4. Francotte, E.; Lindner, W. Chirality in Drug Research; WileyVCH: Weinheim, Germany, 2006.

5. Meyers, R. A. Molecular Biology and Biotechnology: A Comprehensive Desk Reference; Wiley-VCH: 1995.

6. Amemiya, R.; Yamaguchi, M. Org. Biomol. Chem. 2008, 6, 26.

7. Katz, T. J. Angew. Chem. Int. Ed. 2000, 39, 1921.

8. Morrison, D. J.; Trefz, T. K.; Piers, W. E.; McDonald, R.; Parvez, M. J. Org. Chem. 2005, 70, 5309.

9. Botek, E.; Champagne, B. J. Chem. Phys. 2007, 127, 204101.

10. Rajca, A.; Miyasaka, M. In Functional Organic Materials, Syntheses, Strategies and Applications; Wiley-VCH: Weinheim, Germany, 2007; pp 547.

11. Botek, E.; André, J.; Champagne, B.; Verbiest, T.; Persoons, A. J. Chem. Phys. 2005, 122, 234713.

12. Norel, L.; Rudolph, M.; Vanthuyne, N.; Williams, J. A. G.; Lescop, C.; Roussel, C.; Autschbach, J.; Crassous, J.; Racau, R. Angew. Chem. Int. Ed. 2009, 49, 99.

13. Alkorta, I.; Blanco, F.; Elguero, J.; Schröder, D. Tetrahedron: Asymmetry 2010, 21, 962.

14. Abbate, S.; Bazzini, C.; Caronna, T.; Fontana, F.; Gambarotti, C.; Gangemi, F.; Longhi, G.; Mele, A.; Sora, I. N.; Panzeri, W. Tetrahedron 2006, 62, 139.

15. Urbano, A. Angew. Chem. Int. Ed. 2003, 42, 3986.

16. Sahasithiwat, S.; Mophuang, T.; Menbangpung, L.; Kamtonwong, S.; Sooksimuang, T. Synth. Met. 2010, 160, 1148.

17. Cramer, C. J. Essentials of Computational Chemistry: Theories and Models; John Wiley and Sons: UK, 2004.

18. Hobza, P.; Müller-Dethlefs, K. Non-Covalent Interactions: Theory and Experiment; Royal Society of Chemistry: UK, 2009.

19. Zhao, Y.; Truhlar, D. G. J. Phys. Chem. A 2006, 110, 13126.

20. Yanai, T.; Tew, D. P.; Handy, N. C. Chem. Phys. Lett. 2004, 393, 51.

21. Zhao, Y.; Truhlar, D. G. Theor. Chem. Acc. 2007, 120, 215.

22. Zhao, Y.; Truhlar, D. G. Acc. Chem. Res. 2008, 41, 157.

23. Zhao, Y.; Truhlar, D. G. J. Chem. Theory Comput. 2008, 4, 1849.

24. Ahlrichs, R.; Penco, R.; Scoles, G. Chem. Phys. 1977, 19, 119.

25. Grimme, S. J. Comput. Chem. 2004, 25, 1463.

26. Grimme, S. J. Comput. Chem. 2006, 27, 1787.

27. Grimme, S.; Antony, J.; Schwabe, T.; Muck-Lichtenfeld, C. Org. Biomol. Chem. 2007, 5, 741.

28. Mackie, I. D.; DiLabio, G. A. J. Phys. Chem. A 2008, 112, 10968.

29. Moellmann, J.; Grimme, S. Phys. Chem. Chem. Phys. 2010, 12, 8500 .

30. Murguly, E.; McDonald, R.; Branda, N. R. Org. Lett. 2000, 2, 3169.

31. Carrillo, R.; López-Rodríguez, M.; Martín, V.; Martín, T. Angew. Chem. Int. Ed. 2009, 48, 7803.

32. Yamaguchi, M.; Okubo, H.; Hirama, M. Chem. Commun. 1996, 1771.

33. Honzawa, S.; Okubo, H.; Anzai, S.; Yamaguchi, M.; Tsumoto, K.; Kumagai, I. Bioorg. Med. Chem. 2002, 10, 3213.

34. Neese, F. ORCA -- an Ab Initio, Density Functional and Semiempirical Program Package, Version 2.6.35, University of Bonn, 2008.

35. Weigend, F.; Ahlrichs, R. Phys. Chem. Chem. Phys. 2005, 7, 3297.

36. Weigend, F.; Häser, M.; Patzelt, H.; Ahlrichs, R. Chem. Phys. Lett. 1998, 294, 143.

37. Schwabe, T.; Grimme, S. Phys. Chem. Chem. Phys. 2007, 9, 3397.

38. Boys, S. F.; Bernadi, F. Mol. Phys. 1970, 19, 553.

39. Xu, Y.; Zhang, Y. X.; Sugiyama, H.; Umano, T.; Osuga, H.; Tanaka, K. J. Am. Chem. Soc. 2004, 126, 6566. 